\title{
Analysis of differentially expressed microRNA of TNF- $\alpha$-stimulated mesenchymal stem cells and exosomes from their culture supernatant
}

\author{
Hualin $\mathrm{Ma}^{1}$, Shuyan Zhang ${ }^{1}$, Ying X $\mathrm{u}^{2}$, Rongrong Zhang ${ }^{1}$, Xinzhou Zhang ${ }^{1}$
}

\begin{abstract}
${ }^{1}$ Department of Nephrology, Shenzhen People's Hospital, Shenzhen Key Laboratory of Kidney Disease, Second Clinical Medical College, Jinan University, Shenzhen, China 2Department of Hematology, Shenzhen People's Hospital, Second Clinical Medical College, Jinan University, Shenzhen, China
\end{abstract}

Submitted: 2 July 2017

Accepted: 6 September 2017

Arch Med Sci 2018; 14, 5: 1102-1111

DOI: https://doi.org/10.5114/aoms.2017.70878

Copyright @ 2017 Termedia @ Banach

\section{Abstract}

Introduction: To analyze the microRNA expression of tumor necrosi factor $\alpha$ (TNF- $\alpha$ ) stimulated mesenchymal stem cells (MSCS) and exosomes from their culture supernatant.

Material and methods: TNF- $\alpha(20 \mathrm{ng} / \mathrm{ml})$ was used to stimulate MSCs, which were then regarded as TNF- $\alpha$ cells (TC), while unstimulated cells were the normal control cells (NCC). MSCs and their culture supernatant were harvested after $48 \mathrm{~h}$. Subsequently, exosomes were isolated from culture supernatants with ExoQuick-TC and were divided into two groups, TNF- $\alpha$ exosomes (TE) and normal control exosomes (NCE). Then, the microRNAs were measured by high-throughput sequencing and the results were differentially analyzed. Finally, the correlation of the target genes corresponding to differently expressed microRNAs was analyzed by gene ontology (GO) and KEGG pathway analysis.

Results: High-throughput sequencing showed that the cellular compartment (TC vs. NCC) had 280 microRNAs. miR-146a-5p was a uniquely up-regulated microRNA $(p<0.001)$ and the most significantly down-regulated microRNA among the 279 microRNAs included was miR-150-5p ( $p<0.001)$. There were 180 differentially expressed microRNAs in the exosome compartment (TE vs. NCE), where miR-146-5p ( $p<0.001)$ was one of 176 upregulated microRNAs and miR-203b-5p ( $p<0.001)$ was one of 4 downregulated microRNAs. Coincidentally, bioinformatics analysis showed that IRAK1 was a critical target gene of miR-146-5p related to the Toll-like receptor (TLR) signaling pathway. Conclusions: In contrast with the control group, there were significantly differentially expressed microRNAs in both MSCs and exosomes. Interestingly, miR-146a-5p was up-regulated in both comparative groups, and its target gene IRAK1 plays a crucial part in the TLR signaling pathway. These investigations demonstrate a new direction for subsequent inflammation mechanistic studies.

Key words: tumor necrosis factor $\alpha$, mesenchymal stem cell, exosomes, microRNA.

\section{Introduction}

Mesenchymal stem cells (MSCs) have the potential to differentiate and impact important immune regulation, tissue repair, etc. [1-5]. Some of these

\author{
Corresponding author: \\ Xinzhou Zhang \\ Department \\ of Nephrology \\ Shenzhen People's Hospital \\ Shenzhen Key Laboratory \\ of Kidney Disease \\ Second Clinical \\ Medical College \\ Jinan University \\ 1017 Dongmen \\ North Road \\ 518020 Shenzhen, China \\ Phone: + 86-0755-25533018- \\ 3500 \\ E-mail: xin.zhou@medmail. \\ com.cn
}


approaches have been applied in clinical practice. However, there have been some problems, such as limited differentiation potential, and the fact that MSCs may transform into tumor cells or improve tumor progression [6]. Moreover, the survival rate of MSCs in injured tissues was low. It is believed that MSCs can repair damaged tissue mainly by paracrine signaling [7]. However, the specific mechanism needs further investigation. In 2010, Lai et al. [8] isolated nutritional factors that had reparative effects and first confirmed exosomes from mesenchymal stem cells (MSC exosomes). MSC exosomes are homologous vesicles secreted in physiological or pathological conditions, with a diameter of approximately 30-100 $\mathrm{nm}$. Exosomes treat diseases by delivering specific proteins, mRNA, and microRNA to target tissue. Mesenchymal stem cells can secrete more exosomes than other sources [9]. An increasing number of studies have reported that the protective effects of MSCs after pretreatment, such as with conditioned media, ischemia-reperfusion [10], hypoxia induction [11], and inflammatory factors, became more pronounced. Because MSCS secreted more bioactive substances after pretreatment [12], the conditioned medium can also simulate MSCs to protect cells and tissues and regulate immunity and inflammation [13].

Tumor necrosis factor $\alpha$ is an activator of a variety of immune cells. Tumor necrosis factor $\alpha$ can increase the immune regulation of MSCs in vitro and in vivo [14]. The culture medium can reduce the inflammation after stimulating MSCs with TNF- $\alpha$ [15]. The culture medium can simulate the biological function of MSCS after being pretreated by TNF- $\alpha$ and may transfer biological information through exosomes as a carrier, but its mechanism has not been elucidated.

In this study, we used TNF- $\alpha$ as an inducible factor in human mesenchymal stem cells. Cells and exosomes were collected, and RNA of cells and exosomes was extracted to detect microRNAs by high-throughput sequencing. The differential expression of microRNAs in the cells and exosomes was analyzed, and subsequent analysis with an elaborate bioinformatics strategy, including gene ontology (GO) analysis and pathway analysis, was performed.

\section{Material and methods}

\section{Ethics statement}

The experiments were approved by the Ethics Committee of the Second Clinical Medical College of Jinan University.

\section{Preparation of HuMSCs}

Human umbilical cord mesenchymal stem cells (HuMSCs) were isolated from human cord tissue using a method described previously [16]. With the written consent of the parents, fresh human umbilical cords were obtained. Wharton's jelly was diced into cubes of approximately $0.5 \mathrm{~cm}^{3}$ and poured into a centrifuge tube. The tissue was treated with collagenase type II (Sigma, USA) at $37^{\circ} \mathrm{C}$ for $8 \mathrm{~h}$, and the resulting solution was filtered. After the mesenchymal tissue was digested and poured into a 200 mesh filter, the filtrate was diluted with PBS, and then the diluted liquid was centrifuged at $2000 \mathrm{rpm}$ for $5 \mathrm{~min}$. After removal of the supernatant fraction, the cells were resuspended in PBS. The resuspended liquid was centrifuged again at $2000 \mathrm{rpm}$ for $5 \mathrm{~min}$. After removal of the supernatant fraction again, the precipitate (mesenchymal tissue) was resuspended in Dulbecco's modified Eagle's medium (Gibco, Carlsbad, CA, USA) with $10 \%$ fetal bovine serum and cultured at $37^{\circ} \mathrm{C}$ in an incubator containing $5 \% \mathrm{CO}_{2}$.

\section{Cell identification and differentiation studies}

To verify the cultured cells, the $3^{\text {rd }}$-passage cells $\left(1 \times 10^{5}\right)$ were suspended in $50 \mathrm{ml}$ of PBS containing $20 \mathrm{ng} / \mathrm{ml}$ fluorescein isothiocyanate (FITC)-coupled antibodies against CD29, CD34, CD45, CD90, CD105, and HLA-DR (Biolegend, San Diego, CA, USA) and FITC-coupled nonspecific IgG as an isotype control (Biolegend, San Diego, CA, USA).

\section{Stimulation of human mesenchymal stem cells by TNF- $\alpha$}

The cells were washed twice with Hank's Balanced Salt Solution (HBSS) to remove serum when MSCs were grown to $80-90 \%$ confluence. Then, the cells were divided into the following groups: 1) normal control cells: without intervention; 2) TNF- $\alpha$ cells: with stimulant alone, $48 \mathrm{~h}$ TNF- $\alpha$ $(20 \mathrm{ng} / \mathrm{ml})$. After $48 \mathrm{~h}$ incubation, supernatants were harvested for isolation of exosomes.

\section{Isolation of exosomes with ExoQuick-TC}

MSC-derived exosomes were isolated using ExoQuick-TC according to the manufacturer's protocol. In brief, cell culture supernatants were collected and centrifuged at 3,000 $\mathrm{g}$ for $15 \mathrm{~min}$ to remove cells and cell debris. Two $\mathrm{ml}$ of ExoQuick-TC Exosome Precipitation Solution was added to $10 \mathrm{ml}$ of the supernatants and the mixture was refrigerated overnight (at least $12 \mathrm{~h}$ ). The ExoQuick-TC/biofluid mixture was centrifuged at $1,500 \mathrm{~g}$ for $30 \mathrm{~min}$ and the supernatants were aspirated. Then, the residual solution was centrifuged at $1,500 \mathrm{~g}$ for $5 \mathrm{~min}$ and removed. The exosome pellet was resuspended in the appropriate buffer for protein or RNA analysis. 


\section{Characterizations of exosomes}

The quality of exosomes was confirmed by dynamic light scattering using a particle and molecular size analyzer (ZetasizerNano ZS, Malvern Instruments) according to the manufacturer's instructions. The quantity of exosomes was determined by the Micro-BCA assay (Pierce, Rockford, IL) for measurement of total protein.

\section{RNA isolation of MSCs}

Total RNAs from HuMSCs were extracted with the mirVana microRNA isolation kit (Life Technologies, Gaithersburg, MD, USA) according to the manufacturer's protocol. RNA integrity was assessed with electrophoresis.

\section{Isolation of RNA from exosomes}

Exosome supernatants were added to $40 \mathrm{pM}$ synthetic cel-miR-39 (UCACCGGGUGUAAAUCAGCUUG) to control and normalize the efficiency of RNA extraction and then transferred to RNase-free tubes for RNA isolation using an miRNeasy Mini Kit (Qiagen, Valencia, CA, USA) according to the manufacturer's protocol. The RNA samples were washed twice in $500 \mu \mathrm{l}$ of RPE buffer and eluted in RNase-free water. The isolated RNA was measured using a NanoDrop 1000 ultraviolet spectrophotometer (Thermo Fisher Scientific) and analyzed by reverse transcription polymerase chain reaction (RT-PCR), followed by quantitative PCR ( $q P C R$ ).

\section{microRNA deep sequencing analysis}

To prepare for deep sequencing, four small RNA libraries were constructed using the Small RNA Expression Kit (Applied Biosystems, Foster City, (A, USA) according to the manufacturer's protocol. Briefly, RNA samples were hybridized and ligated with an adapter mix. Then, reverse transcription was performed, followed by RNase $\mathrm{H}$ digestion and PCR amplification. The PCR products were then size-selected and purified. Each pool of libraries included three biological repeats. Then, the SOLID V2 sequencing system (Applied Biosystems, Foster City, CA, USA) was performed to generate read counts of nucleotide sequences. The sequencing results were analyzed by the SOLID system small RNA analysis pipeline tool (RNA2MAP). The raw data were first filtered to remove low-quality reads. Then the data were mapped to GenBank (http://www.ncbi.nlm.nih. gov/genbank), and sequences matching rRNAs, tRNAs, snoRNAs and scRNAs were discarded. Finally, by mapping to the miRBase database (http://www.mirbase.org), known and novel microRNAs were identified. The total read counts of each sample were normalized to reads per million
(RPM). To perform differential expression analysis, a DEGseq package tool [17] was applied and the significantly differential microRNAs were identified according to the $p$-value $(<0.05)$.

\section{Bioinformatics analysis}

GO analysis and pathway analysis were used a method that we described previously [18].

\section{Target gene analysis of microRNA expression profiles}

To analyze target genes repressed by microRNAs, we used a previously developed method. Repression scores for individual target genes were calculated according to the formula below. Briefly, the TargetScan Database provides an individual microRNA's repression rate for each of its target genes. By combining the repression rates of all related microRNAs, we can calculate the effect of the entire microRNA profile based on the repression rate of one specific target. Importantly, this method takes into consideration the abundance of each microRNA. Therefore, the repression score represents the effect of the microRNA profiles on a specific target. The significantly differential targets were further identified using DEGseq $(p<0.05)$. To identify microRNAs corresponding to each pathway, target genes were analyzed by the miRWalk tool [19]. Only significantly expressed microRNAs were identified.

\section{Results}

\section{Characterization of MSCs}

HuMSCs were generated by standard procedures in ex vivo culture. HuMSCs were morphologically defined by a fibroblast-like appearance (Figure 1). Flow cytometric analysis of passage 3 cells confirmed that cells were negative for CD34, CD45 and HLA-DR and that cells were positive for CD29, CD90 and CD105.

\section{Characterization of MSC-Exos}

Transmission electron microscopy clearly revealed that MSC-Exos exhibited a cup-shaped or roundshaped morphology with a diameter of 30-100 nm. The detection of origin immunoblotting indicated that the MSC-Exos expressed exosomal markers such as CD81 and CD63 (Figure 2).

\section{microRNA profiles of MSCs}

Using a microRNA deep sequencing method, we investigated the microRNA expression changes of MSCs in the TNF- $\alpha$ cell group and control cell group. High-throughput raw sequencing reads were generated by the SOLiD sequencing system. As a result, 36,842,471 (TC group) and 15,483,812 

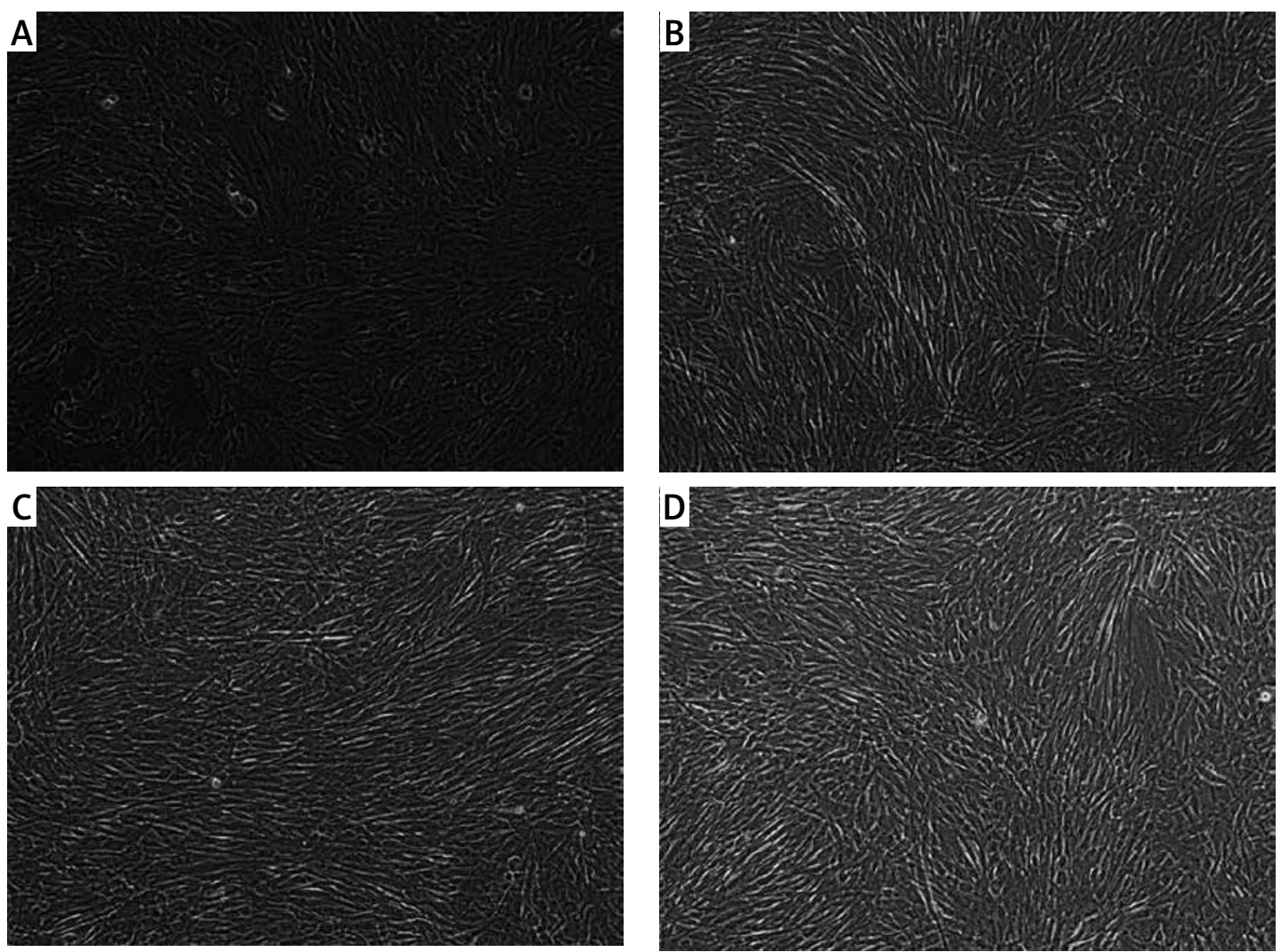

Figure 1. Image of HuMSCs observed by electron microscopy (original magnification $\times 100$ ). A - Control-HuMSCs growth state at 24 h, B - TNF- $\alpha$ HuMSCs growth state at 24 h, C - control HuMSCs growth state at 48 h, D - TNF- $\alpha$ HuMSCs growth state at $48 \mathrm{~h}$

(NC group) reads were obtained. Compared with the GenBank and Rfam databases, we found $13,205,775$ and 4,389,656 microRNA fragments, respectively. We then sought to investigate the TNF- $\alpha$ stimulation-related changes in microRNAs. The DEGseq package tool identified differentially expressed genes based on the Poisson distribution. Using DEGSeq, we performed a differential comparison of the microRNA expression patterns of the TC group MSCs and NC MSCs. To filter out the non-significantly altered microRNAs, we limited the $p$-value to $p<0.01$. As a result, $280 \mathrm{mi}$ -
croRNAs were identified as the most differentially expressed microRNAs in the TNF- $\alpha$ cell group, including 1 up-regulated microRNA and 279 downregulated microRNAs (Figure 3, Table I), of which only miR-146a-5p was up-regulated, 1.92 times greater than in the control group, while hsa-miR150-5p, hsa-miR-1273-c, hsa-miR-1247-5p and hsa-miR-1208 were down-regulated.

\section{microRNA profiles of exosomes}

Using a microRNA deep sequencing method, we investigated the microRNA expression chang-
A

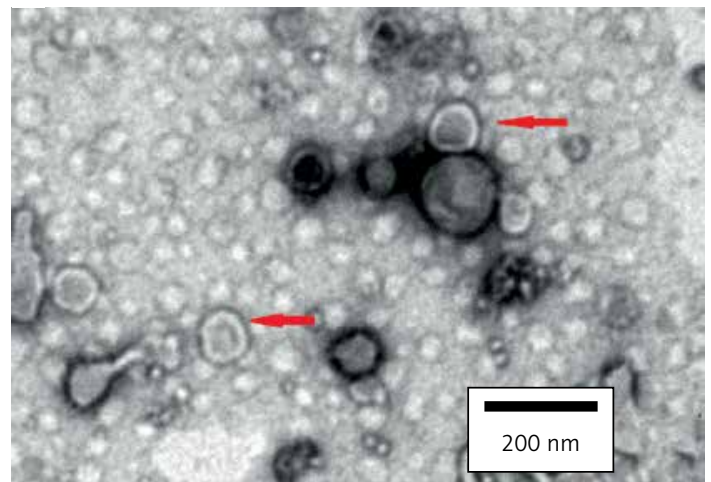

B

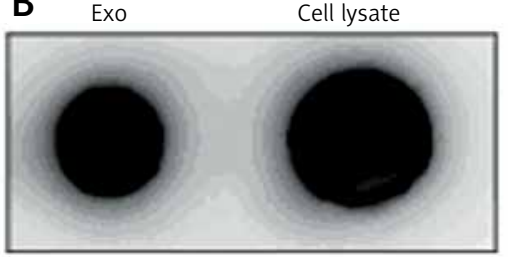

CD63

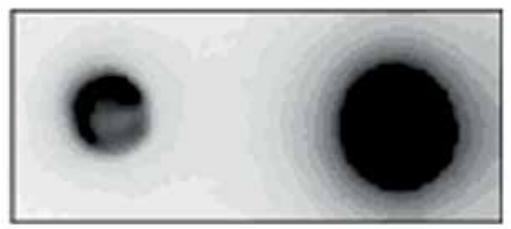

Figure 2. Characterization of exosomes from culture supernatant of MSCs. A - Exosomes at the measurement of $30 \mathrm{~nm}$ to $100 \mathrm{~nm}$ in an electron microscope; B - surface marker CD63/CD81 of positive exosomes and HuMSCs 


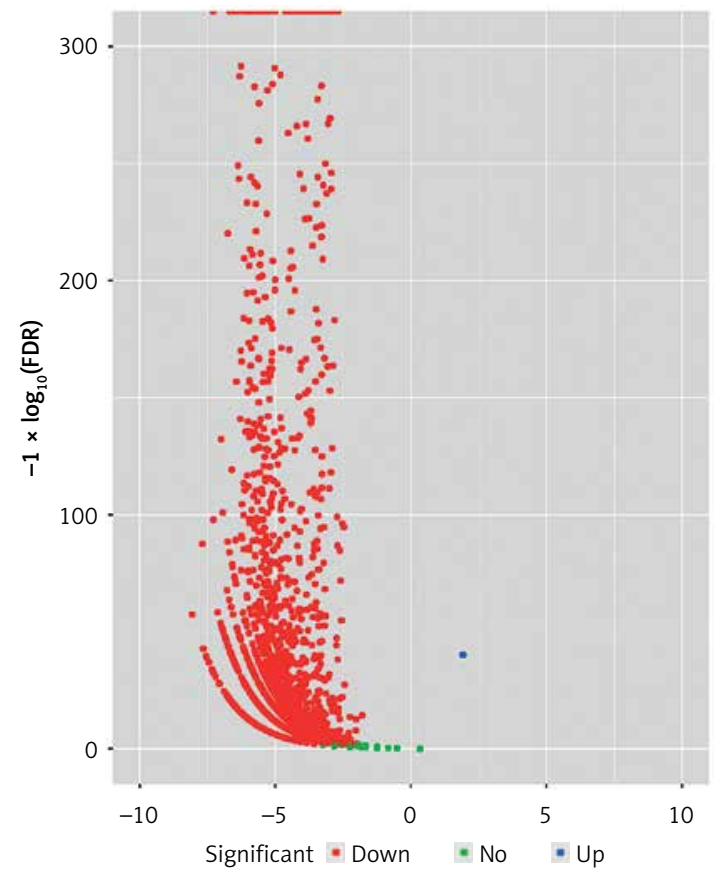

Figure 3. TNF- $\alpha$ cell vs. control cell microRNAs in a volcano plot. The differentially expressed microRNAs of TE compared to NCE group in a volcano plot; each dot represents one microRNA; the blue dots indicate the upregulated microRNAs; the red dots indicate the downregulated microRNAs; the green ones have no significant difference es of MSCs in the TE group and in the NE group. High-throughput raw sequencing reads were generated by the SOLiD sequencing system. As a result, 10,569,238 (TE group) and 15,454,239 (NE group) reads were obtained. Compared with the GenBank and Rfam databases, we found 14,739,30 and 631,271 microRNA fragments, respectively. We then sought to investigate the TNF- $\alpha$-related changes in microRNAs. The DEGseq package tool identified differentially expressed genes based on the Poisson distribution. Using DEGSeq, we performed a differential comparison of the microRNA expression patterns of the TE group exosomes and NE group exosomes. To filter out the non-significantly altered microRNAs, we limited the $p$-value to $p<0.01$. As a result, 180 microRNAs were identified as the most differentially expressed microRNAs in TE group exosomes, including 176 upregulated microRNAs and 4 downregulated microRNAs (Figure 4, Table II). The expression of miR-146-5p, miR-193b-5p, miR-193a-5p and miR-382-5p was up-regulated the most. The expression of miR-203b-5p, miR-203a-3p, miR$1273 \mathrm{~h}-3 \mathrm{p}$, and miR-4540 was down-regulated.

\section{$\mathrm{GO}$ annotation and analysis of the differences}

The cell and exosome genes characterized in the study were evaluated based on their molec-

Table I. The most significantly expressed microRNAs: 1 up-regulated and 19 down-regulated microRNAs

\begin{tabular}{|c|c|c|c|c|}
\hline \multirow[t]{2}{*}{ microRNA ID } & \multicolumn{2}{|c|}{ Relative count (TPM) and group } & \multirow[t]{2}{*}{ Fold change } & \multirow[t]{2}{*}{$P$-value } \\
\hline & TC group & NC group & & \\
\hline hsa-miR-146a-5p & 19.9 & 5.25 & 1.92 & $<0.001$ \\
\hline hsa-miR-150-5p & 4.65 & 477.64 & -6.68 & $<0.001$ \\
\hline hsa-miR-1273c & 1.03 & 94.41 & -6.51 & $<0.001$ \\
\hline hsa-miR-1247-5p & 2.91 & 255.07 & -6.45 & $<0.001$ \\
\hline hsa-miR-1208 & 8.17 & 703.83 & -6.43 & $<0.001$ \\
\hline hsa-miR-378h & 2.78 & 201.65 & -6.18 & $<0.001$ \\
\hline hsa-miR-5693 & 4.9 & 338.22 & -6.11 & $<0.001$ \\
\hline hsa-miR-767-5p & 4.41 & 302.93 & -6.11 & $<0.001$ \\
\hline hsa-miR-4792 & 1.06 & 65.47 & -5.95 & $<0.001$ \\
\hline hsa-miR-2392 & 2.89 & 167.52 & -5.86 & $<0.001$ \\
\hline hsa-miR-4651 & 2.23 & 122.32 & -5.78 & $<0.001$ \\
\hline hsa-miR-718 & 1.36 & 73.24 & -5.75 & $<0.001$ \\
\hline hsa-miR-4315 & 1.06 & 55.5 & -5.71 & $<0.001$ \\
\hline hsa-miR-8069 & 1.06 & 53.75 & -5.66 & $<0.001$ \\
\hline hsa-miR-6075 & 1.47 & 73.56 & -5.65 & $<0.001$ \\
\hline hsa-miR-208b-3p & 4.49 & 211.36 & -5.56 & $<0.001$ \\
\hline hsa-miR-4668-5p & 4.41 & 207.41 & -5.56 & $<0.001$ \\
\hline hsa-miR-4540 & 2.91 & 134.5 & -5.53 & $<0.001$ \\
\hline hsa-miR-4294 & 10.72 & 494.09 & -5.53 & $<0.001$ \\
\hline hsa-miR-4518 & 2.34 & 107.75 & -5.52 & $<0.001$ \\
\hline
\end{tabular}

TPM - transcripts per million. 
ular function, biological process and cellular component annotations. As shown in Figure $5 \mathrm{~A}$, the most enriched cellular components were organelle parts and organelles in cells. In contrast, the cell part is the largest in the exosome genes, as shown in Figure $6 \mathrm{~A}$. The largest group in the distribution of the various functional cell categories is proteins with roles in catalytic activity, as shown in Figure $5 \mathrm{~B}$. The molecular function of exosomes shown in Figure 6 B. Proteins related to cellular and multicellular organismal processes, which comprise the largest group in the distribution of biological processes of cells and exosomes, are shown in Figures $5 \mathrm{C}$ and $6 \mathrm{C}$, respectively.

\section{Signaling pathway analyses}

As described in detail previously [18], we mapped differentially modified genes to the KEGG pathway database using GenMAPP v2.1 and then performed a statistical test to identify enriched metabolic pathways. miR-146a-5p was significantly up-regulated in both the cell group and exosome group. There were 53 target genes in the database that had a corresponding metabolic pathway. There were seven target genes that were closely related to inflammation immunity in nine signaling pathways (Table III), while the Toll-like receptor (TLR) signaling pathway was the most widely distributed (Figure 7).

\section{Discussion}

Many studies have shown that MSCs and MSC exosomes significantly affect kidney diseases [20, $21]$, cardio and pulmonary diseases [22, 23], limb ischemic injury [24] and other diseases. The use of LPS [13], TNF- $\alpha$ [25-28], hypoxia injury [10] and other pretreatments can regulate cell phenotype, secretion of soluble factors and exosomes [29] to play protective roles. Later studies found that exosomes had corresponding target microRNAs that protect

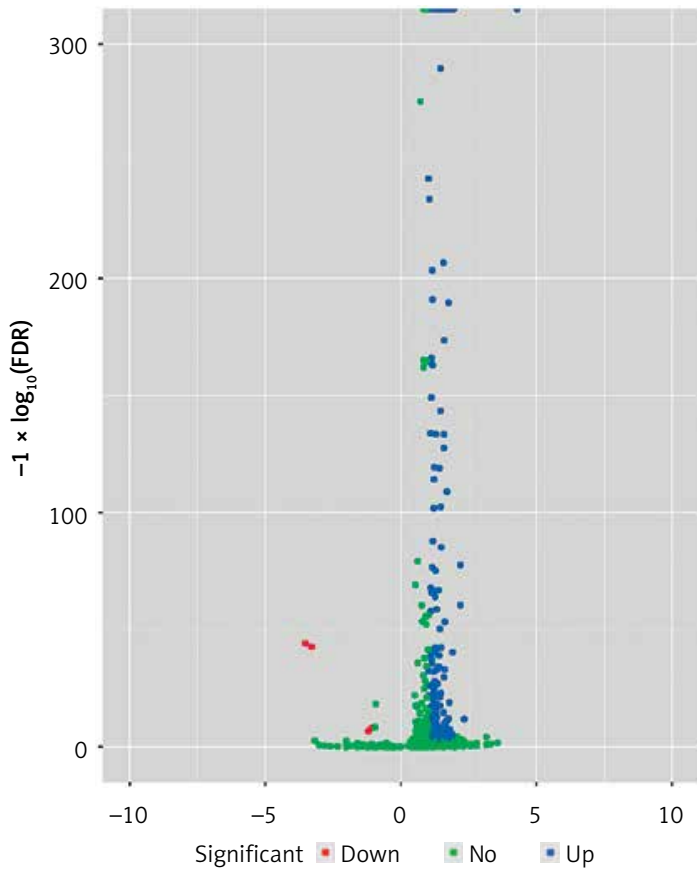

Figure 4. TE vs. NCE microRNAs in a volcano plot. The differentially expressed microRNAs of TE compared to NCE group in a volcano plot; each dot represents one microRNA; the blue dots indicate the upregulated microRNAs; the red dots indicate the downregulated microRNAs; the green ones have no significant difference

the heart [30], inhibit tumor growth [31], enhance tumor chemosensitivity [32], etc. Exosomes may even become a marker to diagnose disease and assess the prognosis of diseases [33]. However, cultured inflammatory-stimulated stem cells can enhance the anti-inflammatory effects [15], which is in contrast with our traditional understanding that inflammatory factors can only cause tissue damage.

It is generally believed that TNF- $\alpha$ is a pro-inflammatory factor that will lead to an inflammatory response. In fact, there are reports [14] that appropriate concentrations of TNF- $\alpha$ do not affect

Table II. The most significantly expressed microRNAs: 4 up-regulated and 4 down-regulated microRNAs

\begin{tabular}{|lcccc|}
\hline microRNA ID & \multicolumn{2}{c}{ Relative count (TPM) and group } & \multirow{2}{*}{ Fold change } & P-value \\
\cline { 2 - 3 } & TE group & NE group & & \\
\hline hsa-miR-146a-5p & 836.23 & 43 & 4.28 & $<0.001$ \\
\hline hsa-miR-382-5p & 321 & 83.54 & 2.34 & $<0.001$ \\
\hline hsa-miR-193a-5p & 63.04 & 13.78 & 2.20 & $<0.001$ \\
\hline hsa-miR-193b-5p & 9.18 & 1.82 & 2.19 & $<0.001$ \\
\hline hsa-miR-203b-5p & 1.89 & 21.72 & -3.52 & $<0.001$ \\
\hline hsa-miR-2036a-5p & 2.27 & 22.3 & -3.29 & $<0.001$ \\
\hline hsa-miR-1273h-3p & 5.87 & 13.5 & -1.20 & $<0.001$ \\
\hline hsa-miR-4540 & 8.71 & 18.56 & -1.09 & $<0.001$ \\
\hline
\end{tabular}


A
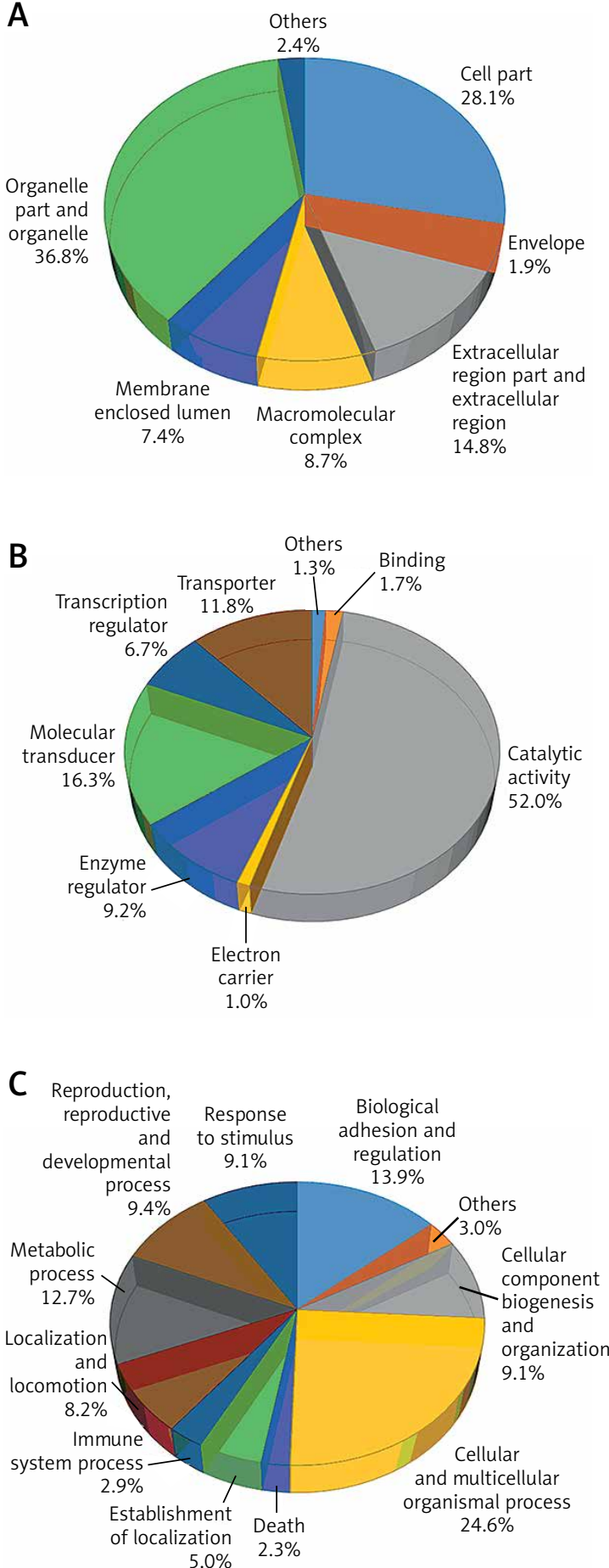

Figure 5. Distribution of significantly differentially expressed microRNAs of cells in the cellular component, molecular function and biological process with high-throughput sequencing

the biological characteristics of cells. In this study, the final concentration of TNF- $\alpha$ was $20 \mathrm{ng} / \mathrm{ml}$, which was consistent with the biological characteristics and growth of the cells. Moreover, we found that TNF- $\alpha$ did not cause cell necrosis, accelerated apoptosis, morphological changes, etc. MicroRNAs are endogenous non-coding small
A

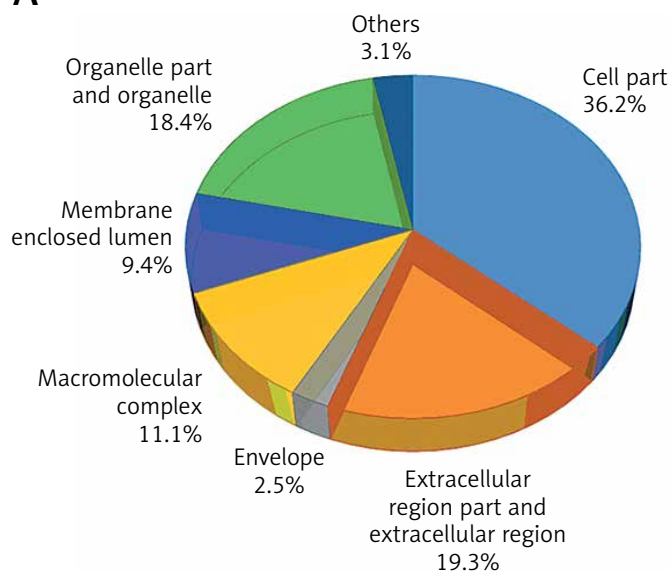

B
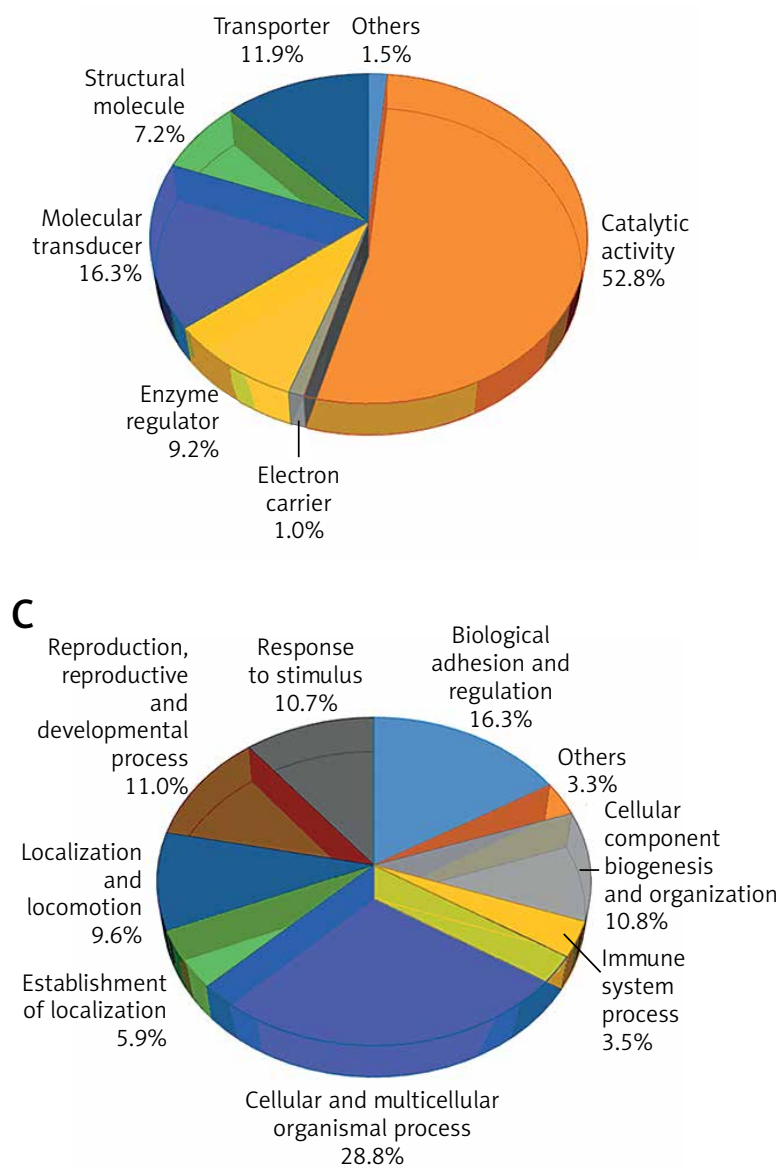

Figure 6. Distribution of significantly differentially expressed microRNAs of exosomes in the cellular component, molecular function and biological process with high-throughput sequencing

RNAs that play an important regulatory role in cells and even tissue biology processes. Therefore, the differential expression of microRNAs and their signaling pathways in cells after stimulation by inflammatory factors can provide important information about pro-inflammatory factors that can also inhibit the inflammatory response. 
Table III. Distributions of the main target gene of miR-146a-5p in the KEGG pathway

\begin{tabular}{|lc|}
\hline Pathway & \begin{tabular}{c} 
Target gene \\
\hline $\begin{array}{l}\text { TOLL_LIKE_RECEPTOR_SIGNALING_ } \\
\text { PATHWAY }\end{array}$
\end{tabular} $\begin{array}{c}\text { TRAF6, IRAK1, } \\
\text { CCL5 }\end{array}$ \\
\hline NF-KB_SIGNALING_PATHWAY & TRAF6, IRAK1 \\
\hline WNT_SIGNALING_PATHWAY & CXXC4, WNT3 \\
\hline CYTOKINE_RECEPTOR_INTERACTION & CNTF \\
\hline JAK_STAT_SIGNALING_PATHWAY & CNTF \\
\hline CHEMOKINE_SIGNALING_PATHWAY & CCL5 \\
\hline $\begin{array}{l}\text { NOD_LIKE_RECEPTOR_SIGNALING_ } \\
\text { PATHWAY }\end{array}$ & CCL5 \\
\hline TGF_BETA_SIGNALING_PATHWAY & RBL1 \\
\hline HEDGEHOG_SIGNALING_PATHWAY & WNT3 \\
\hline
\end{tabular}

In this study, we investigated the mechanism of pro-inflammatory factors on MSCs during ex vivo expansion based on microRNA deep sequencing methods. We identified the significantly altered microRNAs in response to TNF- $\alpha$. Among them, we found 280 microRNAs as the most differentially expressed microRNAs in the TC group, including 1 up-regulated microRNA and 279 down-regulated microRNAs. We also found 180 microRNAs as the most differentially expressed microRNAs in the TE group exosomes, including 176 upregulated microRNAs and 4 downregulated microRNAs.

A number of studies [34-36] have suggested that miR-146a-5p is a microRNA that is closely related to inflammatory immune regulation. Up-reg- ulation of miR-146a-5p has an inhibitory effect on inflammatory immune responses. Moreover, miR-146a-5p is related to important inflammatory pathways, such as NF- $\kappa B$ and Toll-like receptors. Up-regulation of miR-146a-5p was 3.76 and 19.45 times higher in the TC group and TE group compared to the NC group and NE control group, respectively, suggesting that appropriate pro-inflammatory cytokines can change anti-inflammatory microRNAs such as miR-146a-5p and increase anti-inflammatory effects. Recent studies in vitro and in vivo have shown that [34, 37-40] exosomes can inhibit endotoxin-induced inflammatory immune responses via miR-146-5p. In this study, the expression of miR-146-5p in the exosomes group was significantly higher than that of the cell group, which may be due to the simple structure of exosomes and the decreased expression of microRNA. Meanwhile, we believe that the use of exosomes as a small unit may be more effective and efficient because exosomes are smaller and more flexible than cells in terms of biological characteristics. Exosomes are measured in nanometers, so they have a quantitative advantage. In addition, miR-146a-5p can inhibit the transfer of kidney clear cell carcinoma [41], improve allergic rhinitis [42], promote chondrocyte phagocytosi [43], enhance differentiation of spermatogonia [44] and delay senile dementia [35].

KEGG analyses showed that miR-146a-5p was significantly upregulated in both the cell and exosome groups. There were 53 target genes in the database that had corresponding metabolic pathways. There were seven target genes that were

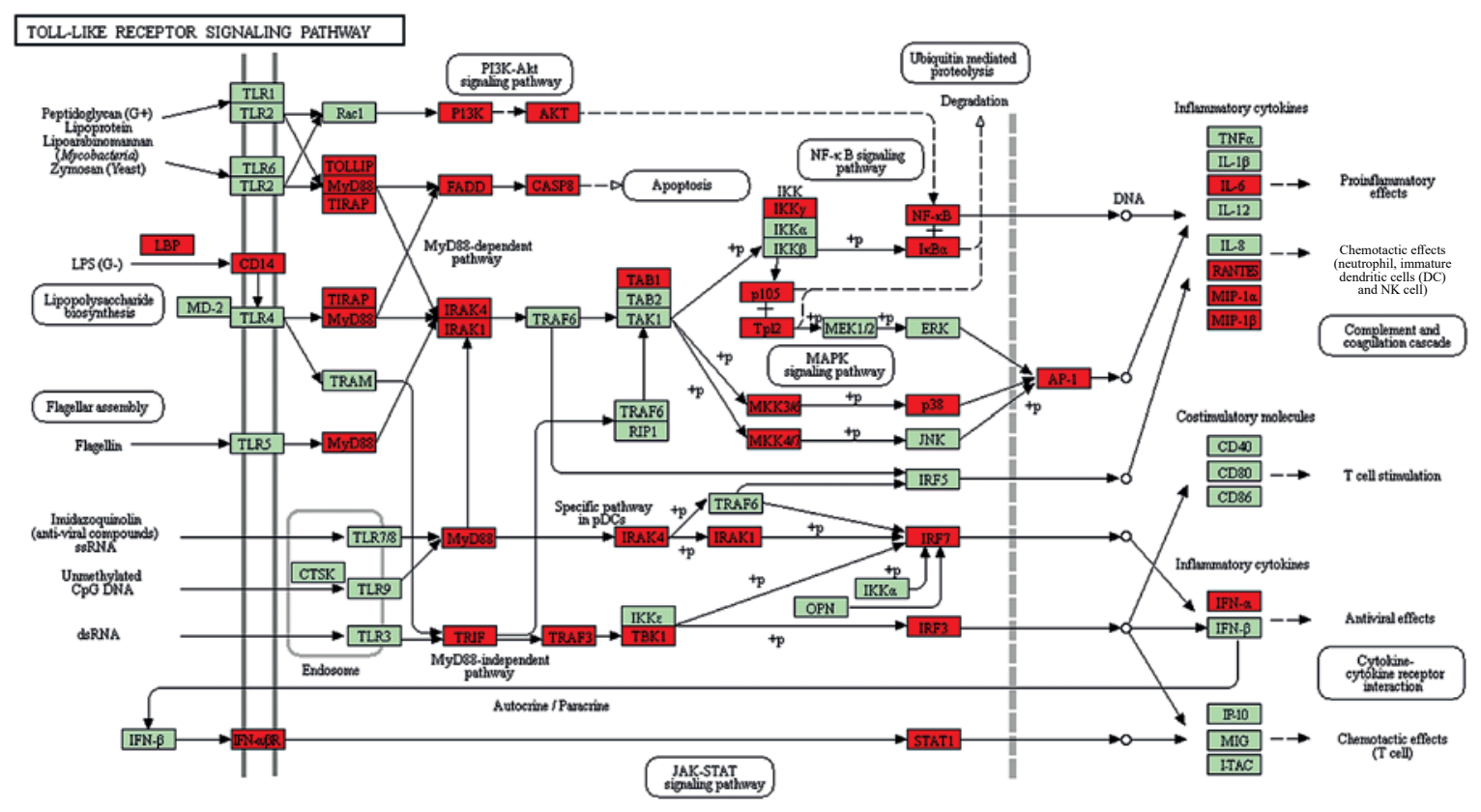

Figure 7. Gene network of miR-146a-5p in the Toll-like receptor signaling pathway 
closely related to inflammation immunity in nine signaling pathways. Some studies reported that miR-146a-5p participates in a variety of biological processes, such as inflammatory immune responses, and that the target genes TRAF6, IRAK1, and CCL5 are involved in the biological effects of multiple metabolic pathways, while the TLR signaling pathway was the most widely distributed. We selected the TLR metabolic pathway to analyze and showed that the IRAK gene played an important role in this metabolic pathway. The miR-146a$5 p$ target gene IRAK1 is the junction of the TLR metabolic pathway and the downstream NF- $\kappa B$ metabolic pathway. Because miR-146a-5p has a negative regulatory immune response, up-regulation of miR-146a-5p may inhibit the transcription of the target gene IRAK1 and inhibit the next level of the inflammatory response.

In summary, the target gene IRAK1 of miR$146 a-5 p$ is involved in the biological process of the TLR metabolic pathway, which is an important inflammatory metabolic pathway. TLR4 can be seen as a major microRNA that regulates the TLR metabolic pathway. miR-146a-5p may provide a new direction for a follow-up study and clinical prevention and treatment of inflammation-related diseases.

In conclusion, we have successfully constructed an experimental model of TNF- $\alpha$ to stimulate MSCs and extracted small RNAs from cells and exosomes. MicroRNA-146a-5p was up-regulated in both the cell group and exosomes group, and the expression ratio was the most obvious. According to bioinformatics analysis, we found that the most important target gene was IRAK1 for microRNA-146a-5p and was involved in the TLR metabolic pathway. miR-146a-5p and IRAK1 may provide a new direction for a follow-up study and clinical prevention and treatment of inflammation-related diseases.

\section{Acknowledgments}

This study was financially supported by the Shenzhen Science and Technology Innovation Committee (grant no. JCYJ20160422151707152).

\section{Conflict of interest}

The authors declare no conflict of interest.

\section{References}

1. Jang MJ, Kim HS, Lee HG, et al. Placenta-derived mesenchymal stem cells have an immunomodulatory effect that can control acute graft-versus-host disease in mice. Acta Haematol 2013; 129: 197-206.

2. Jia Z, Jiao C, Zhao S, et al. Immunomodulatory effects of mesenchymal stem cells in a rat corneal allograft rejection model. Exp Eye Res 2012; 102: 44-9.
3. Gu F, Molano I, Ruiz P, Sun L, Gilkeson GS. Differential effect of allogeneic versus syngeneic mesenchymal stem cell transplantation in MRL/lpr and (NZB/NZW)F1 mice. Clin Immunol 2012; 145: 142-52.

4. Franquesa M, Hoogduijn MJ, Bestard O, Grinyo JM. Immunomodulatory effect of mesenchymal stem cells on B cells. Front Immunol 2012; 3: 212.

5. De Miguel MP, Fuentes-Julian S, Blazquez-Martinez A, et al. Immunosuppressive properties of mesenchymal stem cells: advances and applications. Curr Mol Med 2012; 12: 574-91.

6. Ilmer M, Vykoukal J, Recio Boiles A, Coleman M, Alt E. Two sides of the same coin: stem cells in cancer and regenerative medicine. FASEB J 2014; 28: 2748-61.

7. Yin $\mathrm{H}$, Jiang $\mathrm{H}$. Application prospect of stem cell-derived microvesicles in regeneration of injured tissues. J Biomed Engineering 2015; 32: 688-92.

8. Lai RC, Arslan F, Lee MM, et al. Exosome secreted by MSC reduces myocardial ischemia/reperfusion injury. Stem Cell Res 2010; 4: 214-22.

9. Yeo RW, Lai RC, Zhang B, et al. Mesenchymal stem cell: an efficient mass producer of exosomes for drug delivery. Adv Drug Deliv Rev 2013; 65: 336-41.

10. Li L, Jin S, Zhang Y. Ischemic preconditioning potentiates the protective effect of mesenchymal stem cells on endotoxin-induced acute lung injury in mice through secretion of exosome. Int J Clin Exp Med 2015; 8: 3825-32.

11. Overath JM, Gauer S, Obermuller N, et al. Short-term preconditioning enhances the therapeutic potential of adipose-derived stromal/stem cell-conditioned medium in cisplatin-induced acute kidney injury. Exp Cell Res 2016; 342: 175-83.

12. Cao H, Hui Q, Yan Y, et al. Pretreatments with injured microenvironmental signals altered the characteristics of human umbilical cord mesenchymal stem cells. Biotechnol Lett 2016; 38: 157-65.

13. Ti D, Hao H, Tong C, et al. LPS-preconditioned mesenchymal stromal cells modify macrophage polarization for resolution of chronic inflammation via exosome-shuttled let-7b. J Transl Med 2015; 13: 308.

14. Crisostomo PR, Wang Y, Markel TA, Wang M, Lahm T, Meldrum DR. Human mesenchymal stem cells stimulated by TNF-alpha, LPS, or hypoxia produce growth factors by an NF kappa B- but not JNK-dependent mechanism. Am J Physiol Cell Physiol 2008; 294: C675-82.

15. Su W, Wan Q, Huang J, et al. Culture medium from TNF-alpha-stimulated mesenchymal stem cells attenuates allergic conjunctivitis through multiple antiallergic mechanisms. J Allergy Clin Immunol 2015; 136: 423-32 e8.

16. Yan-Wu G, Yi-Quan K, Ming L, et al. Human umbilical cord-derived Schwann-like cell transplantation combined with neurotrophin-3 administration in dyskinesia of rats with spinal cord injury. Neurochem Res 2011; 36: 783-92.

17. Wang L, Feng Z, Wang X, Zhang X. DEGseq: an R package for identifying differentially expressed genes from RNAseq data. Bioinformatics 2010; 26: 136-8.

18. Zhang X, Ma H, Huang J, Dai Y. Characterization of the phosphoproteome in SLE patients. PLoS One 2012; 7: e53129.

19. Dweep H, Gretz N. miRWalk2.0: a comprehensive atlas of microRNA-target interactions. Nat Methods 2015; 12: 697.

20. Zhu XY, Lerman A, Lerman LO. Concise review: mesenchymal stem cell treatment for ischemic kidney disease. Stem Cells 2013; 31: 1731-6.

21. Gatti S, Bruno S, Deregibus MC, et al. Microvesicles derived from human adult mesenchymal stem cells pro- 
tect against ischaemia-reperfusion-induced acute and chronic kidney injury. Nephrol Dial Transplant 2011; 26: 1474-83.

22. Angelini F, Ionta V, Rossi F, et al. Exosomes isolation protocols: facts and artifacts for cardiac regeneration. Front Biosci (Schol Ed) 2016; 8: 303-11.

23. Aliotta JM, Pereira M, Li M, et al. Stable cell fate changes in marrow cells induced by lung-derived microvesicles. J Extracell Vesicles 2012; 1: 10.3402/jev.v1i0.18163.

24. Li X, Liu L, Chai J. Progress of mesenchymal stem cell-derived exosomes in tissue repair. Chin J Reparat Reconstruct Surg 2015; 29: 234-8.

25. Hu C, Han Z, Yang Z, Li L, Luo W, Ji Y. TNF-alpha enhances hematopoiesis-supportive effect of conditioned culture medium from human umbilical cord-derived MSCs in vitro. Chin J Pathophysiol 2013; 29: 1679-84.

26. Xiao Q, Wang SK, Tian H, et al. TNF-alpha increases bone marrow mesenchymal stem cell migration to ischemic tissues. Cell Biochem Biophys 2012; 62: 409-14.

27. Heo SC, Jeon ES, Lee IH, Kim HS, Kim MB, Kim JH. Tumor necrosis factor-alpha-activated human adipose tissue-derived mesenchymal stem cells accelerate cutaneous wound healing through paracrine mechanisms. J Invest Dermatol 2011; 131: 1559-67.

28. Zhu H, Jiang XX, Guo ZK, et al. Tumor necrosis factor-alpha alters the modulatory effects of mesenchymal stem cells on osteoclast formation and function. Stem Cells Dev 2009; 18: 1473-84.

29. Lee C, Mitsialis SA, Aslam M, et al. Exosomes mediate the cytoprotective action of mesenchymal stromal cells on hypoxia-induced pulmonary hypertension. Circulation 2012; 126: 2601-11.

30. Lu H, Buchan RJ, Cook SA. MicroRNA-223 regulates Glut4 expression and cardiomyocyte glucose metabolism. Cardiovasc Res 2010; 86: 410-20.

31. Katakowski M, Buller B, Zheng X, et al. Exosomes from marrow stromal cells expressing miR-146b inhibit glioma growth. Cancer Lett 2013; 335: 201-4.

32. Lou G, Song X, Yang F, et al. Exosomes derived from miR-122-modified adipose tissue-derived MSCs increase chemosensitivity of hepatocellular carcinoma. J Hematol Oncol 2015; 8: 122.

33. Spinetti G, Fortunato O, Caporali A, et al. MicroRNA-15a and microRNA-16 impair human circulating proangiogenic cell functions and are increased in the proangiogenic cells and serum of patients with critical limb ischemia. Circ Res 2013; 112: 335-46.

34. Nahid MA, Satoh M, Chan EK. Interleukin 1beta-responsive microRNA-146a is critical for the cytokine-induced tolerance and cross-tolerance to Toll-like receptor ligands. J Innate Immun 2015; 7: 428-40.

35. Gauna AE, Park YJ, Nayar G, et al. Dysregulated co-stimulatory molecule expression in a Sjogren's syndrome mouse model with potential implications by microRNA-146a. Mol Immunol 2015; 68: 606-16.

36. Saba R, Sorensen DL, Booth SA. MicroRNA-146a: a dominant, negative regulator of the innate immune response. Front Immunol 2014; 5: 578.

37. Li M, Wang J, Fang Y, et al. microRNA-146a promotes mycobacterial survival in macrophages through suppressing nitric oxide production. Sci Rep 2016; 6: 23351.

38. Rebane A, Runnel T, Aab A, et al. MicroRNA-146a alleviates chronic skin inflammation in atopic dermatitis through suppression of innate immune responses in keratinocytes. J Allergy Clin Immunol 2014; 134: 83647 e11.
39. Wang L, Wang HC, Chen C, et al. Differential expression of plasma miR-146a in sepsis patients compared with non-sepsis-SIRS patients. Exp Ther Med 2013; 5: 1101-4.

40. Iyer A, Zurolo E, Prabowo A, et al. MicroRNA-146a: a key regulator of astrocyte-mediated inflammatory response. PLoS One 2012; 7: e44789.

41. Wotschofsky Z, Gummlich L, Liep J, et al. Integrated microRNA and mRNA signature associated with the transition from the locally confined to the metastasized clear cell renal cell carcinoma exemplified by miR-146-5p. PLoS One 2016; 11: e0148746.

42. Liu HJ, Zhang AF, Zhao N, Li XZ. Role of miR-146a in enforcing effect of specific immunotherapy on allergic rhinitis. Immunol Invest 2016; 45: 1-10.

43. Zhang F, Wang J, Chu J, et al. MicroRNA-146a induced by hypoxia promotes chondrocyte autophagy through Bcl-2. Cell Physiol Biochem 2015; 37: 1442-53.

44. Huszar JM, Payne CJ. MicroRNA 146 (Mir146) modulates spermatogonial differentiation by retinoic acid in mice. Biol Reprod 2013; 88: 15. 